\title{
Bioelectronics: the promise of leveraging the body's circuitry to treat disease
}

\author{
Flavia Vitale ${ }^{*}, 1,2,3$ \& Brian Litt $1,2,4$ \\ ${ }^{1}$ Center for Neuroengineering \& Therapeutics, University of Pennsylvania, 3320 Smith Walk, 301 Hayden Hall, Philadelphia, PA \\ 19104, USA \\ 2Department of Neurology, University of Pennsylvania, 3320 Smith Walk, 301 Hayden Hall, Philadelphia, PA 19104, USA \\ ${ }^{3}$ Department of Physical Medicine \& Rehabilitation, University of Pennsylvania, 3320 Smith Walk, 301 Hayden Hall, Philadelphia, \\ PA 19104, USA \\ ${ }^{4}$ Department of Bioengineering, University of Pennsylvania, 3320 Smith Walk, 301 Hayden Hall, Philadelphia, PA 19104, USA \\ * Author for correspondence: vitalef@pennmedicine.upenn.edu
}

\begin{abstract}
“'The most effective devices, like early pacemakers, defibrillators and brain stimulators for movement disorders, often start quite simply and then evolve steadily over time, incrementally over generations of devices and patients. In order to succeed on this new frontier, we must be patient, methodical and focused, painstakingly mapping relevant anatomy, making safe, durable devices and materials, developing simple algorithms and establishing clear, unquestionable measures of benefit."
\end{abstract}

First draft submitted: 10 October 2017; Accepted for publication: 16 October 2017; Published online: 14 December 2017

Since 1950, life expectancy has increased at a rate of more than 3 years per decade worldwide. Progress stalled for a while in Europe after the collapse of Soviet Union and in Africa in the 1990s due to the HIV epidemic, but the upward trend has more recently resumed - life expectancy increased globally by 5 years between 2000 and 2015 [1]. Unfortunately, a longer life does not necessarily translate into better health. The World Health Organization predicts that approximately 10 years of our existence will be spent suffering from disease or disability caused by a chronic condition such as asthma, hypertension, a neurologic disorder, inflammatory illness, pain or diabetes [1,2].

The mainstay of traditional therapy for chronic conditions is medications, pharmacological agents that attack the disease on a molecular level. The success of many of these drug-based strategies remains suboptimal, either because the mechanism underlying the disease has not been completely elucidated, or because poor selectivity and side effects render treatment ineffective or unsustainable. Yet, we have known for decades that there is another knob we could turn to control - or maybe completely suppress - the pathogenesis of a number of chronic diseases: the nervous system. Dysfunctions in parasympathetic fibers innervating smooth muscles in the lungs, for example, lead to the airway constriction associated with asthma and worsen other obstructive pulmonary diseases. Similarly, autonomic neural pathways mediate hormone secretion, cardiovascular function, blood pressure and recent studies suggest that the immune response is regulated by specific neuronal circuits [3].

In light of these considerations, neuromodulation emerges as a natural solution for tackling a number of chronic diseases in a way that's different and complementary to pharmacotherapy. The concept is not new: neuromodulation in the central nervous system is a well-established treatment for Parkinson's disease, refractory epilepsy, dystonia, essential tremor and neuropathic pain. Spearheaded by these and vagal nerve stimulation technologies for epilepsy [4] and depression [5], the rapidly expanding field of 'Bioelectronics' is promising to revolutionize clinical care and improve the life of millions of patients worldwide.

The term Bioelectronics has been around since the mid-1990s, when researchers began to make complex electronic devices to interface with the body for a variety of applications [6]. The field has evolved steadily since that time, as complex devices have been deployed in tissues as diverse as bone, brain, the GI tract and heart, and transducers and their components have grown to span a host of novel materials and molecularly engineered devices down to the nanoscale [7]. For the sake of this discussion, however, we will use the term 'Bioelectronics' as defined in an editorial by Famm et al. [8] in 2013 to specifically refer to its applications for neuromodulation. In these applications new devices and technologies are intended to directly modulate activity in dysfunctional neural circuits 
giving rise to disease. In these conditions pathophysiology may be manifested in the brain, peripheral nervous system or other organs whose function is, at least in part, under neural control. Targeted peripheral neuromodulation is already approved for treating urinary incontinence [9], chronic neuropathic pain [10], and sleep apnea [11] and it is being investigated for use in a rapidly expanding list of other conditions, including rheumatoid arthritis [12], Crohn's disease [13], diabetes [14], hypertension [15] and heart failure [16].

\section{Why now? Why has it taken so long?}

Though neural control in chronic diseases outside of the nervous system has been appreciated for many years, technological barriers have prevented scientists from fully leveraging these circuits for therapy. This is rapidly changing. A confluence of recent advances in electronics, materials science and computation are fueling explosive advances in Bioelectronics, catalyzed by major funding efforts from industry and federal agencies. These advances include revolutionary leaps forward in our ability to miniaturize device components [17], flexible, dissolvable and more biocompatible materials, and more efficient, expandable components for computation, power and data transmission. With the confluence of these advances, it is now becoming feasible to realize implantable devices that can stimulate and sense - safely and selectively - activity in individual and bundles of human nerves to effectively treat disease. Basic research laboratories are rapidly catching on to this translational revolution, as evidenced by exciting projects funded by DARPA's 'Electric Prescription program' (ElectRx), NIH's new 'Stimulating Peripheral Activity to Relieve Conditions' (SPARC) and exciting efforts funded by industry partners, such as Glaxo Smith Kline and Google's Alphabet, in the form of Galvani Bioelectronics.

The benefits of this paradigm shift toward bioelectronic therapies, compared to drugs is clear, both in terms of reduced side-effects and long-term, cost reduction. However, there are still major challenges that the bioelectronics community needs to address in order to fully realize the potentials of these technologies.

Delivering safe neuromodulation therapy requires targeting specific axonal tracts that travel closely interleaved with other functional classes of fibers. The devices need to modulate one group without interfering with other afferent and efferent bundles that travel to and from organs in the same nerve tract. The problem is daunting, as scientists try to devise sensors that glean information from tiny fibers that intermix, twist and turn together, while ignoring signals from adjacent fibers may carry vastly different information at different rates to different targets. Furthermore, selectively activating specific neural circuits relies on a clear knowledge of the anatomy of the human peripheral nervous system, and of the functions of individual fibers that branch out from the brain and spinal cord in millions of nerves. Understanding this biological map, which can vary greatly from person to person, and is programmed genetically at birth, is perhaps the greatest challenge currently confronting 'bioelectronic engineers'. Nerves that appear quite discreet and robust as they leave the spinal cord branch, divide and eventually end in what appear to be frayed, microscopic tufts buried in connective tissue on organs, arranged in patterns that in many cases are completely unknown. Even the tools to map these structures at clinically meaningful scales are only now being developed.

Designing efficient stimulation therapies also necessitates understanding the fundamental mechanisms underlying normal and pathological states of the nerves, as well as advancing neural decoding to reconstruct those states from recorded individual and compound action potentials. Present devices employed in clinical use apply relatively large stimulation currents to all fibers in a nerve bundle via comparatively large electrodes encircling nerves. To decode and activate smaller groups of fibers will require novel stimulation strategies, in addition to novel hardware, to leverage differences in depolarization thresholds in nerves of different diameters, function and configuration. Improved neural decoding coupled with machine learning algorithms and closed-loop device architectures will permit paradigms to drive nerve functions to an optimal state - tailored to the individual condition, anatomy and disease - simply by adaptively adjusting the stimulation parameters to the instantaneous neural response or longer downstream measures that may require novel sensors to track new classes of biomarkers. Such devices could require sophisticated computation that may require resources outside of what is currently embodied in implantable devices alone.

Long-term reliability of recorded signals and modulation therapy will demand novel electrode interfaces able to chronically function in harsh physiological environments, while minimally impacting the structural and functional integrity of the implanted tissues. Engineered materials combining softness with enhanced electrochemical properties and advanced fabrication strategies will be key to realizing long-term stable, functional electrode/nerve interfaces. Resistance to inflammation and our native 'foreign body response' will be important to maintaining workable impedance, charge transfer and electrical connectivity. Long-term reliability and safety are also vital 
concerns, as some devices may need to last 25 years in the body or more, depending upon the condition being treated.

Data sampling, transmission, storage and analysis in a closed-loop architecture as well as efficient power management also present significant challenges. Solutions will need to be creative, and may require building dedicated pipelines and infrastructure to link implants, personal digital assistants or mobile phone/computation devices to the internet and the cloud, while ensuring patient safety and privacy.

Addressing all these challenges - and this list is by no means exhaustive - may appear a daunting task. The good news is that today, for the first time, many of these problems appear to be tractable.

With the help of novel mapping modalities at the nanoscale [18], including nanoparticles and genetic tools for imaging and stimulation, neuroscientists are reconstructing the atlas of the visceral nerves and deciphering the wiring diagrams of discrete circuits innervating the peripheral organs down to the resolution of the individual fibers. New understanding of the mechanisms regulating the functions and signaling of those neural circuits, their correlation with disease-specific biomarkers and the development of new animal models have significantly accelerated the pathway from proof-of-concept demonstration to clinical translation. Exquisite innovation from the materials science community is leading to the integration of high performance nanoscale conductors - like carbon nanotubes [19-21] and graphene [22] - into flexible devices with novel electronic and optical properties. Exciting new materials are giving engineers the ability to make active, foldable, high-density neural interfaces that can dissolve after use [23] and novel magnetic nanoparticle-based approaches for wireless neuromodulation in vivo [24].

Large companies like IBM and Google are working on new algorithms and machine learning tools to mine the data generated and transmitted by the implantable devices and new startup companies like Blackfynn are developing dedicated tools for clinical data integration, visualization and analysis. Critical mass is building, and many are awaiting the first powerful proof of principle clinical devices to enter the field.

\section{How is this coming bioelectronic revolution poised to play out?}

Some large companies are placing big bets on the success of bioelectronic medicine. These include traditional companies in the medical device space, such as Medtronic and Boston Scientific, as well as new companies arising from other scientific disciplines, such as data mining and the pharmaceutical industry, companies like Google and GSK. Public and private entities, such as the NIH, DARPA and GSK, are dedicating significant resources to jumpstarting research efforts in this space worldwide [8], with an eye toward building new markets, clinical translation and adoption. Propelled by capital injection and by the discoveries of the scientific community, a growing number of startup companies are proposing new target diseases for neuromodulatory intervention, releasing key preliminary data that further demonstrate the validity of the bioelectronic medicine approach [25] and taking on the risks of early stage technology development.

Thanks to these efforts from the private and public sector, today it is becoming easier to envision new clinical care in scenarios in which patients may have devices implanted to treat chronic hypertension or control their asthma in outpatient doctors' offices or outpatient surgery centers. These patients may be discharged home linked to secure data telemetry and the cloud to allow physicians to monitor effectiveness of the therapy and remotely adjust device parameters.

\section{What does a 'bioelectronic revolution' mean for society?}

Chronic diseases affect large segments of the population, with an increasing societal and economic burden that will only become greater in an ageing society. The bioelectronic revolution promises to improve patient outcome and quality of life, and there is potential that one time implants, if easily constructed and deployed, may be significantly less expensive than chronic medications taken for years. Alongside scientific and technical innovation, researchers and industry need to come up with innovative solutions to make these devices affordable and available to the patients who need them the most, regardless of their income and where they live.

As an example pelvic floor dysfunctions (PFD), such as urinary incontinence and overactive bladder, disproportionally affect women because of greater risk factors related to pregnancy and childbirth [26]. The regional burden of PFDs is greatest and increasing at higher rates in low-resource and developing countries, because of the additional risks due to heavy work, under nutrition, concomitant medical conditions and a greater risk of pelvic floor trauma during childbirth unattended by a healthcare professional. In addition to the pain and discomfort, women affected by PFDs can also be subjected to societal stigma [27]. Evidence indicates that, if accessible, peripheral nerve stimulation for control of urinary functions could significantly reduce the burden of disease for 
these women and profoundly impact the quality of their life. The challenges are straight-forward, but the financial model must make sense in order to maximize impact. Highly expensive devices that require extensive surgery and frequent replacement, will just not be impactful in these sorts of disorders. The same may be true for asthma, which disproportionally affects individuals in groups least able to pay for expensive medical devices. True value of therapies and devices is often reflected. One example is China, where families often pay for devices and treatments out of pocket. In these cases the value proposition must be clear, as it is unlikely that a family would impoverish themselves to pay for therapy that is not proven effective. New insurance and healthcare models, particularly in countries with low average income and insurance rates, may help, but it will be vital to make bioelectronic devices affordable as well as effective. Along with technological innovation comes responsibility to make therapies that have maximal positive impact on mankind, not just a select few who have access because of wealth.

Of course, it is important to realize that as exciting as the term 'bioelectronic medicine' may sound, neuromodulation to treat disease, both centrally and peripherally, has been around for many years, and is an important component of business for some of the world's largest medical device companies. Though many creative investigators are running as fast as they can to develop novel technologies to treat disease, it is important that the steps they take as they progress through the medical device pipeline be deliberate, methodical and that they learn from the successes of these industrial giants. The most effective devices, like early pacemakers, defibrillators and brain stimulators for movement disorders, often start quite simply and then evolve steadily over time, incrementally over generations of devices and patients. In order to succeed on this new frontier, we must be patient, methodical and focused, painstakingly mapping relevant anatomy, making safe, durable devices and materials, developing simple algorithms and establishing clear, unquestionable measures of benefit. Though there is tremendous excitement and investment activity in this area, the field needs simple, straight-forward successes on which to build technology platforms, viable business models and to earn patient, physician and insurer trust. So far the preliminary data are encouraging, but the world is anxiously awaiting the first unequivocal evidence of viable, clinical success using technology a cut above standard industry neurostimulators. Despite investor pressures and good spirited academic competition, it is worth it for us to carefully vet our inventions and be cautious of our claims of success as new results roll in. A new era in medical therapy may be upon us, and it behooves us to usher it in the right way.

\section{Financial \& competing interests disclosure}

The authors wish to acknowledge the support of CURE Taking Flight Award to F Vitale and NIH R01NS099348, NIH U01-NS094340, Mirowski Family Foundation, Neil and Barbara Smit to B Litt. B Litt is a co-founder and has financial interests in Blackfynn. The authors have no other relevant affiliations or financial involvement with any organization or entity with a financial interest in or financial conflict with the subject matter or materials discussed in the manuscript apart from those disclosed.

No writing assistance was utilized in the production of this manuscript.

\section{References}

1 World Health Organization. World Health Statistics 2016. World Health

Organization. www.who.int/gho/publications/world_health_statistics/2016/en/

2 Stuckler D, Siegel K. Sick Societies. Oxford University Press.

https://global.oup.com/academic/product/sick-societies-9780199574407?cc=us\&lang=en\&

3 Pavlov VA, Tracey KJ. Neural regulation of immunity: molecular mechanisms and clinical translation. Nat. Neurosci. 20(2), 156-166 (2017).

4 Handforth A, DeGiorgio CM, Schachter SC et al. Vagus nerve stimulation therapy for partial-onset seizures: a randomized active-control trial. Neurology 51(1), 48-55 (1998).

5 Rush AJ, George MS, Sackeim HA et al. Vagus nerve stimulation (VNS) for treatment-resistant depressions: a multicenter study. Biol. Psychiatry 47(4), 276-286 (2000).

6 Nicolini C. From neural chip and engineered biomolecules to bioelectronic devices: an overview. Biosens. Bioelectron. 10(1-2), 105-127 (1995).

7 Asilomar Bioelectronics Symposium 2017.

https://abs.soe.ucsc.edu/sites/default/files/1st\%20Asilomar\%20Bioelectronics\%20Symposium\%20abstract\%20booklet_1.pdf

8 Famm K, Litt B, Tracey KJ, Boyden ES, Slaoui M. Drug discovery: a jump-start for electroceuticals. Nature 496(7444), 159-161 (2013).

9 Siegel SW, Catanzaro F, Dijkema HE et al. Long-term results of a multicenter study on sacral nerve stimulation for treatment of urinary urge incontinence, urgency-frequency, and retention. Urology 56(6 Suppl. 1), 87-91 (2000). 
10 Deer T, Pope J, Benyamin R et al. Prospective, multicenter, randomized, double-blinded, partial crossover study to assess the safety and efficacy of the novel neuromodulation system in the treatment of patients with chronic pain of peripheral nerve origin. Neuromodulation 19(1), 91-100 (2016).

11 Strollo PJ, Soose RJ, Maurer JT et al. Upper-airway stimulation for obstructive sleep apnea. N. Engl. J. Med. 370(2), 139-149 (2014).

12 Koopman FA, Chavan SS, Miljko S et al. Vagus nerve stimulation inhibits cytokine production and attenuates disease severity in rheumatoid arthritis. Proc. Natl Acad. Sci. USA 113(29), 8284-8289 (2016).

13 Bonaz B, Sinniger V, Hoffmann D et al. Chronic vagus nerve stimulation in Crohn's disease: a 6-month follow-up pilot study. Neurogastroenterol. Motil. 28(6), 948-953 (2016).

14 Malbert C-H, Picq C, Divoux J-L, Henry C, Horowitz M. Obesity-associated alterations in glucose metabolism are reversed by chronic bilateral stimulation of the abdominal vagus nerve. Diabetes 66(4), 848-857 (2017).

15 Ng FL, Saxena M, Mahfoud F, Pathak A, Lobo MD. Device-based therapy for hypertension. Curr. Hypertens. Rep. 18(8), 61 (2016).

16 Kishi T. Deep and future insights into neuromodulation therapies for heart failure. J. Cardiol. 68(5), 368-372 (2016).

17 Nelson BJ, Kaliakatsos IK, Abbott JJ. Microrobots for minimally invasive medicine. Annu. Rev. Biomed. Eng. 12(1), 55-85 (2010).

18 Alivisatos AP, Andrews AM, Boyden ES et al. Nanotools for neuroscience and brain activity mapping. ACS Nano 7(3), 1850-1866 (2013).

19 Vitale F, Summerson SR, Aazhang B, Kemere C, Pasquali M. Neural stimulation and recording with bidirectional, soft carbon nanotube fiber microelectrodes. ACS Nano 9(4), 4465-4474 (2015).

20 Lissandrello CA, Gillis WF, Shen J et al. A micro-scale printable nanoclip for electrical stimulation and recording in small nerves. J. Neural. Eng. 14(3), 036006-036012 (2017).

21 McCallum GA, Sui X, Qiu C et al. Chronic interfacing with the autonomic nervous system using carbon nanotube (CNT) yarn electrodes. Sci. Rep. 7, 11723 (2017).

22 Kuzum D, Takano H, Shim E et al. Transparent and flexible low noise graphene electrodes for simultaneous electrophysiology and neuroimaging. Nat. Commun. 5, 5259 (2014).

23 Yu KJ, Kuzum D, Hwang S-W et al. Bioresorbable silicon electronics for transient spatiotemporal mapping of electrical activity from the cerebral cortex. Nat. Mater. 15(7), 782-791 (2016).

24 Chen R, Romero G, Christiansen MG, Mohr A, Anikeeva P. Wireless magnetothermal deep brain stimulation. Science 347(6229), 1477-1480 (2015).

25 Eisenstein M. Neurodevice startups target peripheral nervous system. Nat. Biotechnol. 31(10), 865-866 (2013).

26 Irwin DE, Kopp ZS, Agatep B, Milsom I, Abrams P. Worldwide prevalence estimates of lower urinary tract symptoms, overactive bladder, urinary incontinence and bladder outlet obstruction. BJU Intl. 108(7), 1132-1138 (2011).

27 Walker GJA, Gunasekera P. Pelvic organ prolapse and incontinence in developing countries: review of prevalence and risk factors. Int. Urogynecol. J. 22(2), 127-135 (2010). 
